\title{
Efficacy and Safety Evaluation of Buyang Huanwu Decoction Combined with Wuling Powder in the Treatment of Deep Vein Thrombosis after Fracture
}

\author{
Kun Ren, Guimei Wei, Yanjie Li, Ruoxing Xing, Hong Cheng \\ Henan Provincial Hospital of Traditional Chinese Medicine, Second Affiliated Hospital of Henan University \\ of Traditional Chinese Medicine, Zhengzhou, Henan, 450008, China
}

Keywords: Buyang huanwu decoction combined with wuling powder, Deep vein thrombosis after fracture, Effect, Safety, Inflammatory factor

\begin{abstract}
Objective: To explore the effect and safety of Buyang Huanwu Decoction combined with Wuling Powder in the treatment of deep vein thrombosis after fracture. Methods: A total of 76 patients with deep vein thrombosis after fracture were selected as the research object, and the enrollment period was from June 2018 to December 2019. Patients in the reference group were randomly divided into a reference group and a study group. Patients in the reference group were treated with conventional western medicine, and the study group was treated with Buyang Huanwu Decoction combined with Wuling Powder on the basis of conventional Western medicine treatment. The curative effect, adverse reactions and inflammatory factor levels after treatment of patients with deep vein thrombosis after fracture were compared between the two groups. Results: The total effective rate of treatment for patients with deep vein thrombosis after fracture in the study group was $100.00 \%$ higher than that of the reference group $86.84 \%$, and the incidence of adverse reactions was $2.63 \%$ lower than that of the reference group $18.42 \%$. Inflammatory factors (IL-6, TNF- $\alpha$, CRP) The level was lower than the reference group, the difference was statistically significant $(\mathrm{P}<0.05)$. Conclusion: The development of Buyang Huanwu Decoction combined with Wuling Powder for the treatment of deep vein thrombosis after fracture can effectively improve the clinical treatment effect and medication safety, and improve the level of inflammatory factors.
\end{abstract}

\section{Introduction}

Deep vein thrombosis after fracture is a common clinical complication. It is mostly caused by thrombus damage caused by fracture and slow blood flow caused by long-term bed rest. Early treatment is needed to avoid serious complications. Clinical treatment of deep venous thrombosis after fracture is often treated with drug anticoagulation and thrombolytic therapy. In recent years, integrated traditional Chinese and western medicine has broadened the mind for clinical disease treatment. Combining traditional Chinese medicine on the basis of conventional Western medicine can improve the overall efficacy [1-3] . This study analyzes the clinical value of Buyang Huanwu Decoction combined with Wuling Powder for treatment of patients with deep vein thrombosis after fracture on the basis of conventional western medicine treatment.

\section{Baseline Information and Methods}

\subsection{Baseline Data}

Seventy-six patients with deep vein thrombosis after fracture were selected as the research object in this hospital, and the enrollment period was from June 2018 to December 2019. Inclusion criteria: (1) Fracture and elective surgical treatment; (2) Diagnosis of deep vein thrombosis after fracture; (3) Patients and their families were aware of the contents of this study and signed informed consent; (4) Approved by the Medical Ethics Committee Approved. Exclusion criteria: (1) Allergy or intolerance to the drugs designed for this study; (2) Severe immune system disease, hematopoietic system disease, endocrine disease; (3) Severe osteoporosis; (4) Malignant tumor or Other serious organ diseases; (5) Cognitive impairment or communication impairment. 
76 patients with deep vein thrombosis after fracture were randomly divided into reference group and study group. Among the 38 patients in the reference group, 21 were male patients and 17 were female patients. The patients were between 22 and 63 years old, with an average age of (48.36 \pm 2.37$)$ years, a duration of $5 \mathrm{~h}-24 \mathrm{~h}$, and an average duration of $(9.65 \pm 2.11)$. $\mathrm{h}$, the number of patients with the right lower limb and the left lower limb were 20 and 18, respectively. Among the 38 patients in the study group, 23 were male patients and 15 were female patients. The patients were 21 to 64 years old, with an average age of $(49.58 \pm 2.31)$ years old, the duration of the disease was 6h-24h, the average duration of the disease was $(10.05 \pm 2.15) \mathrm{h}$, the number of patients with the right lower limb and the left lower limb were 21 and 17, respectively. After comparison, it can be seen that the baseline data of the two groups of patients with deep vein thrombosis after fracture are not significantly different $(\mathrm{P}>0.05)$, and they are clinically comparable.

\subsection{Method}

Patients in the reference group were treated with conventional western medicine, that is, they were instructed to stay in bed, raise the affected limb about $15 \mathrm{~cm}$ above the level of the heart to prevent the affected limb from being compressed or thrombus falling off, and inform the patient of a reasonable and scientific diet. The patient was given low-molecular-weight heparin calcium (Shenzhen Saibaoer Biological Pharmaceutical Co., Ltd.; National Medicine Zhunzi H20060190) subcutaneous injection into the abdominal wall, $0.4 \mathrm{ml} /$ time/d, and continued treatment for 10 days.

The research group was given Buyang Huanwu Decoction combined with Wuling San on the basis of conventional western medicine treatment. The basic prescriptions were: Atractylodes macrocephala, Roasted Astragalus, Chuanxiong, Angelica, Peach kernel, Poria, 12g each, Chuan Achyranthes Bidentata, Red Peony, Red 8g each of flower, Alisma and Adenophora. At the same time, dialectical addition and subtraction treatments are carried out according to the specific conditions of the patients. Among them, patients with poor sleep quality and sleep difficulties are added with $12 \mathrm{~g}$ of Fushen and Albizia bark; for patients with mild pain, $12 \mathrm{~g}$ of Shenqu and fried malt are added; patients with severe pain are added with Frankincense, Corydalis 8g. Decoction in water, $400 \mathrm{ml}$ of juice, 1 dose a day, divided into two doses for 10 days.

\subsection{Evaluation Indicators}

After treatment, the treatment effects of the two groups of patients were evaluated. The efficacy evaluation criteria were as follows: (1) Significantly effective: After treatment, the leg circumference of the affected limb is within $1.5 \mathrm{~cm}$ or the same as the leg circumference of the healthy limb. The color Doppler ultrasound examination results show that the body thrombus disappears and the blood vessels The internal blood flow is smooth and the pain is significantly reduced; (2) Effective: After the treatment, the swelling of the affected limb is significantly relieved, and the pain is significantly improved. The difference between the circumference of the affected limb and the healthy limb is within $2.5 \mathrm{~cm}$, and the color Doppler ultrasound examination results show that part of the thrombus disappeared or blocked the part. Vascular circulation; (3) Ineffective: After treatment, the patient does not meet the above criteria. The total effective rate of treatment $=$ (number of markedly effective cases + number of effective cases) / total number of patients $\times$ $100.00 \%$.

The adverse reactions of the two groups of patients were recorded, such as bleeding, skin necrosis, hematoma, etc.

After the treatment, the levels of inflammatory factors in the two groups of patients were detected, namely interleukin-6 (ie IL-6), serum tumor necrosis factor- $\alpha$ (ie TNF- $\alpha$ ), and C-reactive protein (CRP).

\subsection{Data Processing}

The result data in this article was processed with SPSS21.0, the count data was expressed in the form of $n(\%)$, the comparison of the data between groups was carried out by the c2 test; the measurement data was expressed in the form of ( $\square \square \pm \mathrm{S}$ ), and the t test was used for the data comparison. $\mathrm{p}<0.05$ means that the difference between groups is significant and statistically 
significant.

\section{Results}

\subsection{Comparison of the Curative Effect of Two Groups of Patients with Deep Vein Thrombosis after Fracture}

The total effective rate of treatment of deep vein thrombosis after fracture in the study group was $100.00 \%$ higher than that of the reference group $86.84 \%$, the difference was statistically significant $(\mathrm{P}<0.05)$. See the table below for details.

Table 1 Comparison of Curative Effect between Two Groups of Patients with Deep Vein

Thrombosis after Fracture [n(\%)]

\begin{tabular}{|l|l|l|l|l|}
\hline Gr & Markedly effective & effective & invalid & TTL \\
\hline Reference Gr(n=38) & $15(39.47)$ & $18(47.37)$ & $5(13.16)$ & $33(86.84)$ \\
\hline Research Gr(n=38) & $17(44.74)$ & $21(55.26)$ & $0(0.00)$ & $38(100.00)$ \\
\hline$X^{2}$ & - & - & - & 5.3521 \\
\hline P & - & - & - & 0.0207 \\
\hline
\end{tabular}

3.2 Comparison of Adverse Reactions in Patients with Deep Vein Thrombosis after Fracture in the Two Groups

The adverse reaction rate of patients with deep vein thrombosis after fracture in the study group was $2.63 \%$ lower than that of the reference group $18.42 \%$, the difference was statistically significant $(\mathrm{P}<0.05)$. See the table below for details.

Table 2 Comparison of Adverse Reactions between the Two Groups of Patients with Deep Vein Thrombosis after Fracture [n(\%)]

\begin{tabular}{|l|l|l|l|l|}
\hline Gr & Bleeding & Skin necrosis & hematoma & TTL \\
\hline Reference Gr(n=38) & $2(5.26)$ & $2(5.26)$ & $3(7.89)$ & $7(18.42)$ \\
\hline Research Gr(n=38) & $0(0.00)$ & $0(0.00)$ & $1(2.63)$ & $1(2.63)$ \\
\hline X2 & - & - & - & 5.0294 \\
\hline P & - & - & - & 0.0249 \\
\hline
\end{tabular}

3.3 Comparison of the Levels of Inflammatory Factors in Patients with Deep Vein Thrombosis after Fracture between the Two Groups

The levels of IL-6, TNF- $\alpha$, CRP and other inflammatory factors in patients with deep vein thrombosis after fracture in the study group were lower than those in the reference group, and the difference was statistically significant $(\mathrm{P}<0.05)$. See the table below for details.

Table 3 Comparison of The Levels of Inflammatory Factors in Patients with Deep Vein Thrombosis after Fracture between the Two Groups ( $\bar{\chi} \pm s)$

\begin{tabular}{|l|l|l|l|}
\hline Gr & IL-6(ng/L) & TNF- $\alpha(\mathrm{ng} / \mathrm{L})$ & CRP(mg/L) \\
\hline Reference Gr(n=38) & $13.56 \pm 3.51$ & $20.79 \pm 3.13$ & $5.38 \pm 0.63$ \\
\hline Research Gr(n=38) & $6.27 \pm 1.08$ & $13.05 \pm 3.08$ & $2.56 \pm 0.49$ \\
\hline T & 12.2369 & 10.8653 & 21.7807 \\
\hline P & 0.0001 & 0.0001 & 0.0001 \\
\hline
\end{tabular}

\section{Discussion}

Deep vein thrombosis is a common complication after fracture. It refers to the abnormal aggregation of blood in the deep vein, which affects its return, and eventually forms thrombus, which usually occurs in the lower limbs. Fractures are often accompanied by thrombotic injury, and the patient needs to stay in bed for a long time, which slows down the blood flow of the body and aggravates the formation of deep vein thrombosis of the lower extremities. If not treated in time, it will cause post-thrombotic syndrome, affect the movement of the patient's limbs and hinder the patient Illness recovery [4-7]. 
After clinical treatment of fractures, deep vein thrombosis is usually treated with anticoagulation and thrombolytic therapy, and nutritional support, rehabilitation guidance, etc. are used to relieve the thrombosis. However, the patient will still have a certain degree of lower limb swelling and bleeding after treatment, which affects the disease Recovery. Deep venous thrombosis belongs to "thigh swelling" and "Mai Bi" in the field of traditional Chinese medicine, and it is closely related to the endogenesis of congestion, qi and blood damage, and qi and blood deficiency [8-10]. On the basis of conventional western medicine treatment, TCM treatment with the purpose of replenishing qi and nourishing blood and promoting blood circulation to remove blood stasis can effectively improve clinical efficacy and safety. The results of this article show that the total effective rate of treatment for patients with deep vein thrombosis after fracture in the study group is higher than that of the reference group, the incidence of adverse reactions is lower than that of the reference group, and the level of inflammatory factors is lower than that of the reference group. The difference is statistically significant $(\mathrm{P}<0.05)$. Analysis of the reasons shows that Buyang Huanwu Decoction has the effects of dredging collaterals, invigorating qi, and promoting blood circulation, while Wuling Powder can reduce dampness and diuresis, and Buyang Huanwu Decoction combined with Wuling Powder for treatment of deep vein thrombosis after fracture can promote blood circulation and reduce water consumption Damage to the patient [11-12]. On the basis of conventional western medicine treatment, combined with Buyang Huanwu Decoction and Wulingsan addition and subtraction treatment, it can effectively enhance the body's platelet aggregation, promote thrombolysis, expand blood vessels, improve the body's blood circulation, and improve the level of inflammatory factors in patients [13-14].

To sum up, on the basis of conventional western medicine treatment, the treatment of Buyang Huanwu Decoction combined with Wulingsan addition and subtraction treatment for patients with deep vein thrombosis after fracture has a positive significance in improving the clinical treatment effect, drug safety and improving the level of inflammatory factors. .

\section{References}

[1] Zhou Weijun, Huo Shaochuan, Cai Yingfeng, et al. Yiqi Huoxue Tongluo Decoction combined with aspirin to prevent deep vein thrombosis after artificial hip replacement. Shaanxi Traditional Chinese Medicine, 2020, 41(8): 1070-1073.

[2] Liu Chunxiao, Song Xiuju, Wang Li. Effect of Yiqi Huayu Tongmai Decoction combined with acupoint massage on hemorheology, fibrinogen, D-dimer and inflammatory factors in patients with deep vein thrombosis after femoral neck fracture surgery. Modern Journal of Integrated Traditional Chinese and Western Medicine, 2020, 29(22): 2410-2414.

[3] Bi Fangshan, Yin Tian, Liu Yang, et al. Comparison of the effect of Yiqi Huoxue Decoction and Biefang Xiaozhong Decoction in preventing deep vein thrombosis of the lower limbs after hip fracture surgery in the elderly. China Contemporary Medicine, 2020, 27 (13):154-157.

[4] Hu Junhui. Application effect of Jiawei Buyang Huanwu Decoction in preventing deep vein thrombosis after artificial hip replacement. Journal of Clinical Rational Use, 2020, 13(15): 122-124.

[5] Liu Yingjuan, Fan Bo, Li Xiaoning, et al. Effects of Huoxue Tongmai Decoction on Deep Vein Thrombosis and Coagulation Function in Patients Undergoing Knee Arthroplasty. Thrombosis and Hemostasis, 2020, 26(3): 396-397,401.

[6] Shi Ke, Wen Xinli, Ren Xingmin. The effect of Ziyin Tongluo Decoction aspirin on deep vein thrombosis and coagulation function in patients with stroke. Thrombosis and Hemostasis, 2020, 26(3): 390-391.

[7] Liu Junchang, Zhang Feilong, Pang Kebin. The effect of Jiedu Sanyu Decoction combined with omeprazole on deep vein thrombosis of the lower extremities in patients with pancreatitis. Thrombosis and Hemostasis, 2019, 25(6): 933-934.

[8] Tong Pei, Jiang Hanmei, Di Shi. Observation on the curative effect of Xuefu Zhuyu Decoction 
on preventing deep vein thrombosis after gynecological surgery. Journal of Hubei University of Traditional Chinese Medicine, 2019, 21(5): 61-63.

[9] Qi Lei, Li Huagang, Zhou Yuzhu, et al. Clinical observation of Danshen Huayu Decoction in the treatment of acute deep vein thrombosis of lower extremities. World Latest Medical Information Abstracts (Continuous Electronic Journal), 2020, 20(32) :221-222.

[10] Chen Juan, He Xiaoxiao, Du Yao, et al. The effect of Tongmai Decoction on deep venous thrombosis of closed fractures of lower limbs and the changes of thromboelastogram. World Traditional Chinese Medicine, 2019, 14(8): 2048- 2052.

[11] Shi Ke, Wen Xinli, Ren Shengmin. Ziyin Tongluo Decoction on the formation of deep vein thrombosis of the lower limbs of stroke patients and its effect on the coagulation function of the patients. Thrombosis and Hemostasis, 2019, 25(4): 581- 582.

[12] Zhang Songhong. Evaluation of Taohong Siwu Decoction in the treatment of deep venous thrombosis of lower limbs after femoral intertrochanteric fracture. Reflexology and Rehabilitation Medicine, 2020, 29(2): 40-41.

[13] Hou Haichun. Self-made Quyu Xiaozhong Decoction in the prevention of postoperative deep vein thrombosis in elderly patients with lower limb fractures. Sichuan Traditional Chinese Medicine, 2020, 38(1): 137-139.

[14] Xie Yisong, Yuan Wanfu, Liu Xiaolan, et al. Experimental study on the preventive effect of Xuefu Zhuyu Decoction on deep vein thrombosis in rats. Hunan Journal of Traditional Chinese Medicine, 2019, 35(9): 137-139. 